\title{
Dal genotipo al fenotipo
}

\author{
Giovanni Duro, Carmela Zizzo
}

Centro di Ricerca e Diagnosi Malattie da Accumulo Lisosomiale, Istituto di Biomedicina e Immunologia Molecolare "A. Monroy" (IBIM), Consiglio Nazionale delle Ricerche (CNR), Palermo

\begin{abstract}
From genotype to phenotype
During the last years, genetic tests have been commonly used in clinical practice. Difficult and doubtful diagnoses are frequent and to date many clinicians have needed geneticists for the study of specific genes and gene panels (through next generation sequencing) to reach a reliable diagnosis. The identification of genes and mutations related to a disease, together with the development of faster and cheaper methodologies, has led to a better basic understanding of several diseases, giving clinicians new opportunities for the management of many genetic diseases. However, these important results also showed that genetics is not always conclusive. Indeed, different phenotypic expressions (diseases) are due not only to sequence variants of the genome (mutations). It is increasingly clear that epigenetic factors have an important influence through complex mechanisms of regulation. This field of study is relatively new and the mechanisms involved in the regulation of gene expression are largely unknown.
\end{abstract}

Keywords: Diagnosis, Fabry disease, Genotyping

Nel corso degli ultimi anni la pratica clinica si è avvalsa sempre più frequentemente di test genetici. Molte sono le diagnosi difficili/dubbie e oggi molti clinici chiedono al genetista lo studio di geni specifici e sempre più spesso anche lo studio di pannelli di geni, utilizzando Next Generation Sequencing (NGS), per arrivare a una diagnosi certa (1). L'identificazione di geni e mutazioni correlate a malattia e lo sviluppo di metodi più veloci e meno costosi hanno portato a una migliore comprensione di base di numerose patologie, offrendo al clinico nuove opportunità per la gestione di molte malattie genetiche (2). Ma questi importanti risultati raggiunti hanno anche evidenziato che non sempre la genetica fornisce risposte conclusive. Infatti, molte varianti di sequenza del genoma non spiegano completamente diverse espressioni fenotipiche. L'applicazione di screening di popolazione per

Accepted: June 26, 2017

Published online: July 31, 2017

Indirizzo per la corrispondenza:

Prof. Giovanni Duro

Centro di Ricerca e Diagnosi

Malattie da Accumulo Lisosomiale

Consiglio Nazionale delle Ricerche

Istituto di Biomedicina e Immunologia Molecolare "A. Monroy"

Via Ugo La Malfa, 153

90146 Palermo

giovanni.duro@ibim.cnr.it diverse patologie, ha portato all'identificazione di una quantità enorme di varianti genetiche $(3,4)$. Una variante genetica può essere chiaramente correlata a una malattia e può quindi spiegare il fenotipo di un dato paziente, ma non tutte le varianti genetiche sono responsabili/co-responsabili di una data patologia o coinvolte nella sintomatologia del paziente. Alcune varianti possono essere solo polimorfismi, variabilità individuale, e queste alterazioni potrebbero essere interpretate come fattore di rischio per lo sviluppo di una data malattia. L'epigenetica e fattori ambientali possono influenzare la patogenicità su base individuale (5). È sempre più chiaro che fattori epigenetici hanno un'influenza importante, attraverso meccanismi di regolazione complessi (5). Questo campo di studio è relativamente nuovo e i meccanismi coinvolti nella regolazione dell'espressione genica sono in gran parte sconosciuti. L'interpretazione dei dati genetici è spesso difficile, la patogenicità di una variante e la sua relazione con il fenotipo clinico, in un singolo paziente, spesso restano poco chiare. Queste considerazioni sono particolarmente importanti per molte malattie ereditarie, tra cui le malattie da accumulo lisosomiale. È sicurament e migliorata la comprensione dei diversi fenotipi e una maggiore possibilità di test genetici è oggi disponibile, ma è anche più chiaro che una variante nel relativo gene, non porterà alla manifestazione della malattia in tutti i casi.

Nella Fabry sono state indentificate, ad oggi, circa 900 mutazioni nel gene GLA (http://www.hgmd.cf.ac.uk/ac/gene. php?gene=GLA), ma una percentuale di queste sono classifi- 
cate, dalla comunità scientifica mondiale, varianti genetiche il cui significato è ad oggi sconosciuto (GVUS). Queste mutazioni rappresentano spesso un vero problema per quanto riguarda la consulenza, lo screening familiare, le opzioni di trattamento. Oggi siamo consapevoli che la Fabry potrebbe essere una patologia più complessa di quanto sia ritenuto e i test diagnostici, oggi in uso, non sempre sono sufficienti per confermare la diagnosi clinica. La complessità della malattia è anche testimoniata dalla mancata corrispondenza, in alcuni casi, tra attività enzimatica, manifestazioni cliniche e presenza di accumulo in soggetti che presentano mutazioni nel gene GLA. Probabilmente, come risulta dalla letteratura, nell'insorgenza della malattia potrebbero essere coinvolte anche altre molecole, come: cofattori necessari per la funzionalità dell' $\alpha$ galattosidasi A (per esempio, saposine) (6); recettori per il suo trasporto ai lisosomi (recettore del mannosio-6-fostato e sortilina) (7); proteine che stabilizzano la struttura dell'enzima (chaperones) (8). Tali sistemi dovrebbero essere presi in grande considerazione nello studio/diagnosi della malattia di Fabry. Inoltre potrebbero essere studiati altri geni, correlati alle manifestazioni sistemiche, in cui eventuali mutazioni potrebbero sommarsi con effetto sinergico alle alterazioni nei sistemi implicati nella malattia di Fabry, o comunque nella sintomatologia. Non necessariamente Fabry.

In conclusione possiamo sottolineare che l'identificazione di varianti genetiche oggi non è difficile, ma la loro interpretazione, al contrario, può risultare ancora una sfida. Un approccio standardizzato è auspicabile per affrontare queste difficoltà. Probabilmente lo studio di pannelli di geni (utilizzando NGS) migliorerebbe la conoscenza di molte patologie $(1,9,10)$. Oggi diversi progetti di ricerca sono orientati verso l'identificazione di nuovi marcatori molecolari specifici, per arrivare a una diagnosi certa.

\section{Disclosures}

Financial support: No financial support was received for this submission.

Conflict of interest: The authors have no conflict of interest.

\section{Bibliografia}

1. Lee $H$, Deignan JL, Dorrani N, et al. Clinical exome sequencing for genetic identification of rare Mendelian disorders. JAMA. 2014;312(18):1880-7.

2. Froissart $R$, Piraud $M$, Maire I. Contribution of genotyping in Fabry's disease. Rev Med Interne. 2010;31(Suppl 2): S275-8.

3. Spada M, Pagliardini S, Yasuda M, et al. High incidence of lateronset Fabry disease revealed by newborn screening. Am J Hum Genet. 2006;79(1):31-40.

4. Hwu WL, Chien YH, Lee NC, et al. Newborn screening for Fabry disease in Taiwan reveals a high incidence of the later-onset GLA mutation c.936+919G>A (IVS4+919G>A). Hum Mutat. 2009;30(10):1397-405.

5. Bird A. Perceptions of epigenetics. Nature. 2007;447(7143):396-8.

6. Kase R, Bierfreund $U$, Klein A, et al. Only sphingolipid activator protein B (SAP-B or saposin B) stimulates the degradation of globotriaosylceramide by recombinant human lysosomal alpha-galactosidase in a detergent-free liposomal system. FEBS Lett. 1996;393(1):74-6.

7. Prabakaran T, Nielsen R, Satchell SC, et al. Mannose 6-phosphate receptor and sortilin mediated endocytosis of $\alpha$-galactosidase A in kidney endothelial cells. PLoS One. 2012;7(6):e39975.

8. Yue P, Li Z, Moult J. Loss of protein structure stability as a major causative factor in monogenic disease. J Mol Biol. 2005; 353(2):459-73.

9. Yang $Y$, Muzny DM, Xia F, et al. Molecular findings among patients referred for clinical whole-exome sequencing. JAMA. 2014;312(18):1870-9.

10. Spurdle AB, Healey S, Devereau A, et al; ENIGMA. ENIGMAevidence-based network for the interpretation of germline mutant alleles: an international initiative to evaluate risk and clinical significance associated with sequence variation in BRCA1 and BRCA2 genes. Human Mutation. 2012;33(1):2-7. 